\section{Comparison of Direct Endovascular Treatment Versus Thrombolysis and Rescue-endovascular Treatment for Patients with M1/M2 Occlusion: A Real-life Retrospective Study}

\section{Abstract}

Introduction: Systemic Thrombolysis with tissue Plasminogen Activator (tPA) has been an established therapy for ischemic stroke for more than 20 years, with limited effect on large vessel occlusions. New catheter-based techniques allow high revascularization rates were tPA fails. Systemic thrombolysis remains the standard treatment of care and endovascular treatment is newly established as therapy for large vessel occlusion or rescue therapy after unsuccessful revascularization by thrombolysis. Patients with large vessel occlusions have a very limited reperfusion rate and benefit from direct endovascular treatment (EVT). In this real-life study, patients are compared regarding their outcome after systemic thrombolysis with rescue endovascular treatment in comparison to direct endovascular treatment for large vessel occlusions.

Patients and Methods: Patients are categorized retrospectively by their treatment. Data on 120 patients was used, with the exclusion of 13 patients. Analyses were performed according to mRS and EQ5D after 7 days as well as 10 to 14 weeks. Primary observed outcome was quality of life and mRS after 10 to 14 weeks.

Results: Regarding mRS, initial outcome after 7 days is more successful with direct EVT, but prior thrombolysis shows additional improvement after 10 to 14 weeks. There is no statistically relevant difference in patients that received prior tPA and rescue endovascular treatment in comparison to direct endovascular treatment after 10 to 14 weeks. EQ5D on the other hand shows higher life quality, according to better functional outcome after endovascular treatment. Regarding the primary endpoint of the study, there is no statistically relevant difference between both study groups at the end point of the study.

Discussion: While direct endovascular treatment shows an immediate benefit for patients with large vessel occlusions, over the time course of 10 to 14 weeks there is a higher additional benefit for prior thrombolysis treatment. Regarding that, both treatment arms show a similar outcome. A higher life quality regarding EQ5D is archived after direct EVT while judgement for general ability is rated higher with additional tPA treatment.

Conclusion: In this mono-center study it was shown that patients eligible for thrombolysis with large vessel occlusions should receive tPA if eligible. By lack or insufficient improvement, a rescue endovascular treatment should be applied. Direct endovascular treatment shows a similar outcome regarding $\mathrm{mRS}$ but a higher life quality index.

Keywords: Stroke; Endovascular treatment; Stent retriever; Thrombolysis
Stefan Mausbach ${ }^{1 *}$, Alexandra Gerasimova ${ }^{1}$, Ze'ev Itsekson ${ }^{1}$, Noit Brown ${ }^{1}$, Yvonne Schwammenthal ${ }^{1,2}$, Oleg Merzlyak', Mati Bakon ${ }^{3}$ and David Orion ${ }^{1,2}$

1 Department of Neurology, Sheba Medical Center, Tel HaShomer, Israel

2 Sackler Faculty of Medicine, Tel Aviv, Israel

3 Department of Neuroradiology, Sheba Medical Center, Tel HaShomer, Israel

\section{* Corresponding author:}

Dr. Stefan Mausbach

” S.Mausbach@gmail.com

Department of Neurology, Sheba Medical Center, Tel HaShomer, Israel. .

Tel: 491715234190

Citation: Mausbach S, Gerasimova A, Itsekson Z, Brown N, Schwammenthal $Y$, et al. (2019) Comparison of Direct Endovascular Treatment Versus Thrombolysis and Rescue-endovascular Treatment for Patients with M1/M2 Occlusion: A Real-life Retrospective Study. J Neurol Neurosci Vol.10 No.02:295. 


\section{Introduction}

Ischemic stroke is a disease with rising prevalence. Two-thirds of patients suffer from a cardiovascular incidence from the age of 65 onwards [1]. On the background of aging, specifically in the western world, there is increasing need for effective treatment for ischemic stroke.

Today, there are two main therapeutic approaches. One is thrombolysis by intravenous administration of recombinant tissue Plasminogen Activator (tPA) as systemic therapy, the other is by an endovascular approach. While systemic treatment is limited to a restricted time window, endovascular therapy offers a more extensive window as well as better treatment options for large vessel occlusions where systemic therapy may be limited.

In certain instances, it appears that thrombolytic therapy has limited success. Studies published in 2011 [2] and 2014 [3] showed that thrombolytic therapy has no benefit in large vessels with a clot larger than $8 \mathrm{~mm}$.

Since the approval of the Solitaire FR catheter, stent retriever has become the worldwide standard for endo-vascular treatment. Other catheters, such as Trevo, show similar recanalization rates [4].

In light of these studies, it is important to mention, that endovascular therapy for intracranial artery occlusion is still a second line treatment, according to the latest guidelines of the American Heart Association [5]. The first treatment line for ischemic stroke is still intravenous thrombolysis. Endovascular therapy is an established rescue treatment in those cases where thrombolytic therapy fails or if contraindications to systemic thrombolysis exist.

The aim of this study was to analyze, if there would be a better outcome for direct endovascular treatment in comparison to prior treatment by systemic thrombolysis with no, or insufficient improvement and secondary rescue endovascular treatment in a real-life cohort.

\section{Patients and Methods}

The study protocol was approved by the Committee on Human Research at the Sheba Medical Center. In this retrospective cohort mono-center study, patients were identified from the Database of the Department of Neurology at the Sheba Medical Center. Eligible Patients from January 2014 until December 2017 were included.

\section{Inclusion criteria}

Patients aged $18-90$ years that were diagnosed with an ischemic stroke and occlusion of the middle cerebral artery territory by imaging between January 2014 and December 2017 and received endovascular treatment at Sheba Medical Center, according to the Stroke and Endovascular Unit of Neurology. Included were only patients with a prior mRS of $0-1$.

\section{Exclusion criteria}

Patients not fulfilling the age criteria, improvement after systemic thrombolysis, no indication for mechanical thrombectomy, $\mathrm{mRS} \geq$ 2 , pregnancy, side effects after systemic thrombolysis (bleeding, allergic reaction), basilar occlusion.

\section{Study}

Patients eligible for the study were analysed for medical history and current treatment including blood thinners like platelet antiaggregant, Vitamin-K-Antagonists, NOAC and Heparin. The initial NIHSS and prior mRS were acquired. The treatment was followed by thrombolysis and endovascular treatment in comparison to direct endovascular treatment. Data from CTPerfusion scans was collected and analysed. $m R S \leq 2$ was defined as favorable outcome in comparison to $\mathrm{mRS} \geq 4$ seen as nonfavorable outcome. Consent from all patients to process their data was received.

\section{Questionnaire}

NIHSS and mRS after 7 days were acquired as well as after 10-14 weeks including the EQ5D questionnaire by examination in the ward, follow-up in the outward patient department or telephonic questionnaire.

\section{Statistical analysis}

All data collected in this study was analysed using SPSS version 21.0. Deceased patients during the study were followed up over the national register with day of death. Data was observed using arithmetic mean, medians and standard deviations. The interquartile range was used as a measure of distribution. A comparison between the two groups was performed using crosstables for independent samples and continuous variables.

\section{Results}

55 patients receiving tPA and EVT and 52 patients receiving direct EVT were included in the study (Table 1). A total of 5 where lost for follow up (2 not interested, 3 left the country).

The initial outcome of each procedure was determined by modified Ranking Scale that was evaluated after 7 days (or dismissal) as well as after $10-14$ weeks. mRS $\leq 2$ is seen as favorable outcome as this still holds the potential of regaining ability to carry out all previous activities. $m R S \geq 4$ is seen as nonfavorable outcome as it renders the patient with need for help with own bodily needs and assisted walking, is bed bound or deceased.

There is a first difference after 7 days regarding the two treatment groups (Figure 1). After tPA + EVT, $25 \%$ of all patients reached a favorable outcome while nearly $33 \%$ with direct EVT reached this outcome. Nearly $66 \%$ of patients receiving both treatments show an unfavorable outcome in contrast to $56 \%$ in the EVT group. Important to notice is the higher number of patients with mRS 6 in this group in the first follow up after 7 days.

After 3 months, an increase in mortality in both groups can be seen with a higher rate in the EVT group (Figure 2). Specifically, tPA+EVT shows improvement after 3 months in the groups of $m R S$ 4 and 5 with progress under time and physiotherapy to a better functional outcome and an increase in total of $6 \%$ of favorable 
Table 1 Analysis of study groups regarding age, cardiovascular risk factors, stroke in the past, initial NIHSS and rate of extracranial occlusion in addition to proximal MCA-occlusion.

\begin{tabular}{|c|c|c|c|}
\hline \multicolumn{4}{|c|}{ Baseline characteristics of the study population } \\
\hline \multicolumn{2}{|c|}{ Characteristics } & tPA + EVT $(N=52)$ & EVT $(N=55)$ \\
\hline \multirow{2}{*}{ Age } & Median & 71 & 73.5 \\
\hline & Interquartile range & $34-88$ & $28-90$ \\
\hline Male sex & no. (\%) & 27 (51.9) & $25(45.5)$ \\
\hline \multicolumn{4}{|c|}{ Cardiovascular Risk factors } \\
\hline \multicolumn{2}{|c|}{ Art. Hypertension no. (\%) } & $37(71.15)$ & $35(63.36)$ \\
\hline \multicolumn{2}{|c|}{ Dyslipidemia no. (\%) } & 30 (57.69) & $28(50.91)$ \\
\hline \multicolumn{2}{|c|}{ DM no. (\%) } & $9(17.31)$ & $14(25.45)$ \\
\hline & AF/PAF no. (\%) & $24(46.15)$ & $32(58.18)$ \\
\hline & CHF no. (\%) & $10(19.23)$ & $16(29.09)$ \\
\hline & Stroke in the Past no. (\%) & $8(15.38)$ & $10(18.18)$ \\
\hline & Median HBA1c (\%) & 5.8 & 5.3 \\
\hline \multicolumn{4}{|c|}{ Baseline NIHSS } \\
\hline \multicolumn{2}{|c|}{ median } & 16.5 & 15 \\
\hline Dense Artery sign native CT & median in $\mathrm{mm}$ (no of patients) & 162 mm (37) & $218 \mathrm{~mm}(43)$ \\
\hline Extracranial occlusion & no $(\%)$ & $8(15.3)$ & $9(16.4)$ \\
\hline \multicolumn{4}{|c|}{ Time intubated } \\
\hline \multicolumn{2}{|c|}{ median in days } & 1.55 & 1.4 \\
\hline
\end{tabular}

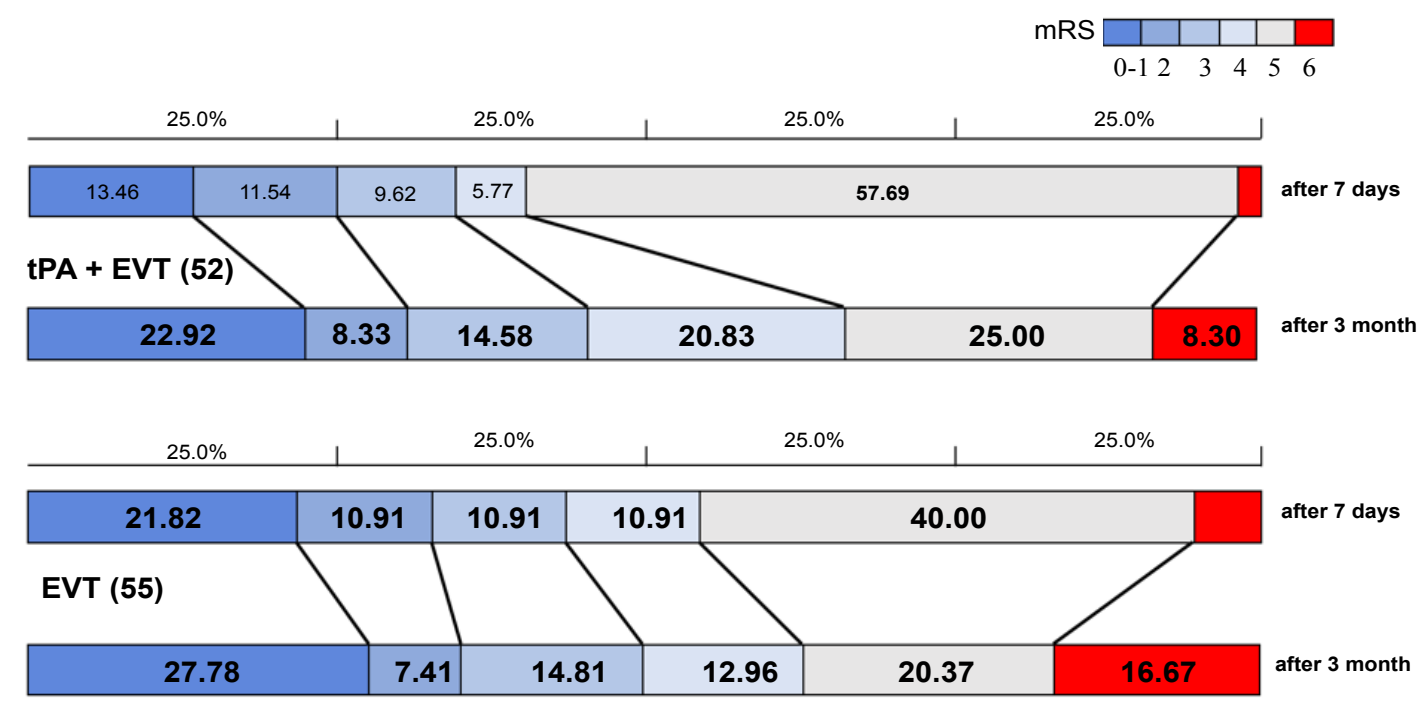

Figure 1 Functional outcome according to mRS after 10-14 weeks. Lines indicate the differences between both treatment arms. mRS 4 to 6 is seen as unfavorable outcome.

outcome. The EVT group shows an increased mortality and only a slight increase in the percentage of favorable outcome (3\%). It should be pointed out that after 3 months, the best possible outcome with mRS 0-1 is highest in the EVT group. Regarding the favorable outcome set to $\mathrm{mRS} \leq 2$, there is no statistical difference between both study arms. The EVT group reaches $35 \%$ in comparison to $31 \%$ in the other group.

The Kaplan-Meier analysis (Figure 2) shows an initial higher rate of death in the first week after treatment in the direct endovascular group. A second decline starts 45 days after EVT treatment and 65 days after prior tPA treatment (Figure 2). In total 20 patients passed away during the study time of 98 days, 3 passed away during the first 7 days after direct EVT, 1 patient after prior tPA treatment.

10 to 14 weeks after the stroke event, patients underwent 2 telephone consultation to the standard EQ5D scale, an instrument of evaluating quality of life and general health status. Patients were questioned regarding their mobility, self-care, general care, pain and depression/anxiety level. The answer options ranged from no influence on life (1), medium influence on life (2) and strong influence on life (3). In the individual analyzes of the different mRS groups, it points out that the EVT group with 
mRS 0-1 has the best ratings (Table 2). From mRS 2 on there is a decline while the subjective ratings stay stable in the TPA+EVT group and only start to decline from mRS3.

Regarding the odds-ratio including all patient outcomes, there is a favor for EVT for mobility and slightly for pain susceptibility, Anxiety and Depression as well as self-care is slightly favored for prior thrombolysis. Outstanding is the result for general ability which clearly favors tPA with additional endovascular treatment (Figure 3).

For further investigation of predefined outcome regarding imaging, patient data were further analyzed for size of penumbra and core infarction (Table 3). Via volume rendering, both could be exactly measured. There is no correlation of penumbra size to outcome. There is slight trend of core infarct size and outcome, yet the position of the core infarction is more predefining then the size.

\section{Discussion}

The treatment by tPA is still the most established treatment for eligible patients. The studies with intra-arterial thrombolysis [6] and early mechanical thrombectomy devices like Merci [7] showed no benefit over tPA. The studies from 2014 and 2015 regarding Stent-Retriever, showed improved outcomes in case of revascularization rate and outcome for large vessel occlusions. The current guidelines by AHA/ASA recommend tPA for patients eligible for the first 4.5 hours after symptom onset. Even if endovascular treatment is considered, tPA is recommended to be given.
Patients received therapy by thrombolysis during the first 4.5 hours after symptom onset if eligible. After lack of improvement, or only minor improvement during the first 45 minutes of thrombolysis treatment, a CTA of head and neck and a CTP were performed. With fitting criteria regarding penumbra and core infarct, patients underwent mechanical thrombectomy during the first $6 \mathrm{~h}$ after symptom onset.

Patients not eligible for thrombolysis treatment following the guidelines by ASA/AHA underwent direct thrombectomy during the first 6 hours of symptoms. The initial analysis regarding $\mathrm{mRS}$ show a better outcome after direct EVT treatment. Nearly 33\% regain the ability to return to normal life including return to work after dismissal from hospital. In comparison, $25 \%$ got the same outcome after prior tPA treatment.

Two-thirds of the TPA+EVT group show an unfavorable outcome which is defined by $m R S \geq 4$ with a very high number of $m R S$ 5 with nearly $58 \%$. After direct EVT treatment, $56 \%$ showed an unfavorable outcome with $40 \%$ of $\mathrm{mRS} 5$, showing in conclusion that more people are rendered with significant loss of life quality after prior thrombolysis.

After 10-14 weeks, there is improvement in both groups. Specifically, in the TPA+EVT group, with improvement specifically for the more severely disabled patients. There is a high shift from mRS 5 to better outcomes and only a slight increase of deceased patients. All in all, 31\% reach a favorable outcome. Direct EVT treatment as well shows a shift to better outcome. 35\% show a $m R S \leq 2$ in the follow up. But the shift from $m R S 5$ points to both directions. The total amount is reduced to $20 \%$ with equal

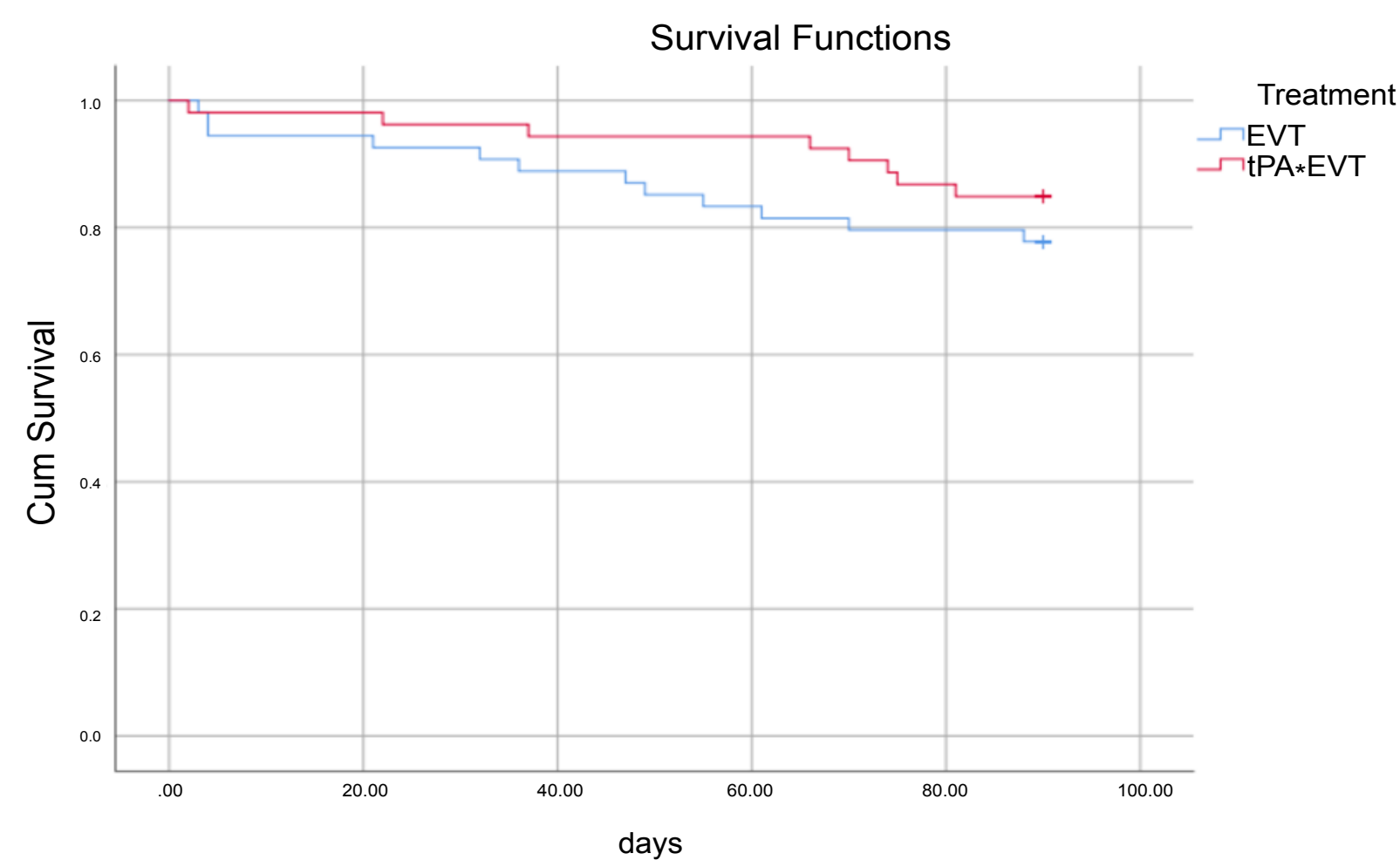

Figure 2 Kaplan-Meier analysis according to both treatment groups with follow up until 90 days. Deceased patients were collected from the national register at the end of the study and the specific day of death could be determined. 
Table 2 Analysis after 10-14 weeks for both treatment groups regarding EQ5D (measurement for general health introduced by European Quality Group in 1990 with a questionnaire of 5 questions). Results are shown in mean value.

\begin{tabular}{|c|c|c|c|c|c|c|c|c|c|c|c|}
\hline \multicolumn{12}{|c|}{ EQ5D Questionnaire after 10-14 weeks } \\
\hline \multicolumn{2}{|c|}{ mRS } & \multicolumn{2}{|l|}{$0-1$} & \multicolumn{2}{|l|}{2} & \multicolumn{2}{|l|}{3} & \multicolumn{2}{|l|}{4} & \multicolumn{2}{|l|}{5} \\
\hline \multicolumn{2}{|c|}{ Treatment arm } & tPA + EVT & EVT & tPA + EVT & EVT & tPA + EVT & EVT & tPA + EVT & EVT & tPA + EVT & EVT \\
\hline \multicolumn{2}{|c|}{ No. Patients } & 14 & 11 & 5 & 3 & 6 & 7 & 9 & 7 & 8 & 9 \\
\hline Mobility & Mean-No. & 1.36 & 1.21 & 1.20 & 2.33 & 1.67 & 2.00 & 2.22 & 2.57 & 3.00 & 2.56 \\
\hline Self-Care & Mean-No. & 1.09 & 1.14 & 1.20 & 2.33 & 1.50 & 2.86 & 2.56 & 3.00 & 3.00 & 3.00 \\
\hline General Ability & Mean-No. & 1.36 & 1.21 & 1.60 & 2.33 & 1.67 & 2.86 & 2.33 & 2.71 & 3.00 & 2.56 \\
\hline Pain & Mean-No. & 1.82 & 1.29 & 1.60 & 1.66 & 1.33 & 1.86 & 1.89 & 2.76 & 1.50 & 1.78 \\
\hline Depression/ Anxiety & Mean-No. & 1.72 & 1.36 & 1.40 & 2.00 & 1.50 & 1.86 & 2.00 & 1.78 & 2.00 & 1.80 \\
\hline
\end{tabular}

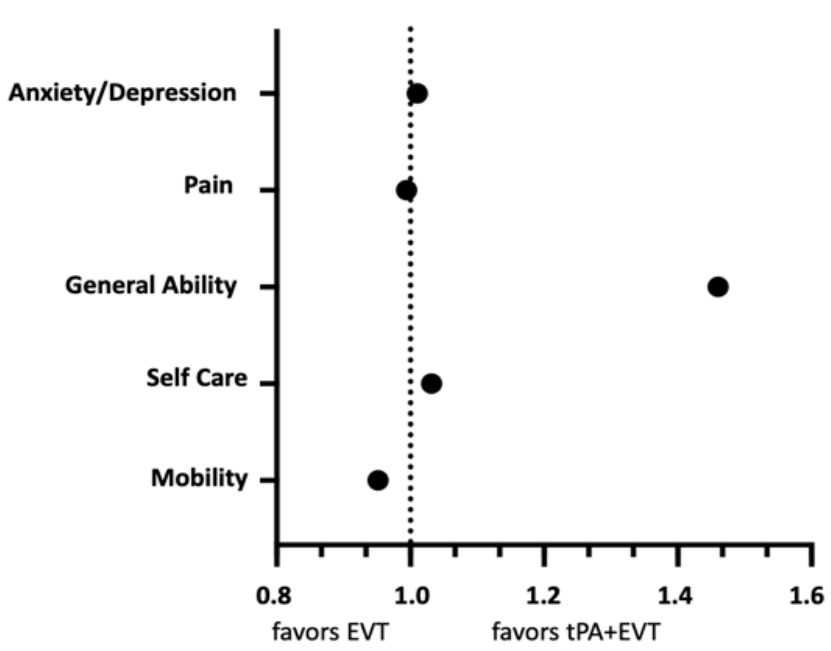

Figure 3 Odds-Ration for both treatment arms throughout mRS 0-5. Favor for direct EVT in Mobility and Pain perception, General Ability favored by prior tPA treatment and Rescue-EVT.

Table 3 Correlation of Penumbra to Core Infarct on CT-Perfusion scans before mechanical thrombectomy. Not statistical relevance can be seen regarding the penumbra and the outcome. There is a slight correlation between size of the core infarction and a higher mRS.

\begin{tabular}{|c|c|c|c|c|c|c|c|}
\hline \multicolumn{7}{|c|}{ CT Perfusion results } \\
\hline mRS & $\mathbf{0}$ & $\mathbf{1}$ & $\mathbf{2}$ & $\mathbf{3}$ & $\mathbf{4}$ & $\mathbf{5}$ & $\mathbf{6}$ \\
\hline Penumbra & 12.92 & 10.92 & 14.21 & 15.06 & 13.72 & 14.95 & 13.81 \\
\hline Core Infarct & 1.08 & 1.39 & 2.26 & 2.49 & 2.64 & 2.06 & 2.44 \\
\hline
\end{tabular}

improvement and worsening. $16,8 \%$ deceased in comparison to $8 \%$ after prior tPA treatment.

The comparison by mRS shows an initial better outcome of direct EVT treatment which favors this method of stroke intervention. $34 \%$ return to a good to very good outcome, there are less patients with unfavorable outcome and there is further improvement in the following 10-14 weeks. Important to notice is the higher rate of death. One reason is unsuccessful revascularization, but also higher rates of intracranial bleedings and massive intracerebral bleedings either due to mechanical injury by catheter or effects by prior anticoagulation or need for loading doses for stent implantations worsen the outcome. Patients underwent endovascular treatment also with elevated INR by Vitamin $\mathrm{K}$ Antagonists or liver disease or treatment by new oral anticoagulations. These agents are still active during the treatment and state a higher bleeding risk. Patients receiving tPA have by definition no bleeding diathesis due to contraindications for treatment. After failure of improvement by tPA, the patient underwent the procedure including CTA and CTP for further diagnostics. On average, the patient underwent $1 \mathrm{~h}$ after TPA the additional endovascular treatment. tPA has a clearance of 550$680 \mathrm{ml} /$ minute from plasma. This results in a half-life $\leq 5$ minutes and a terminal elimination after around 40 minutes. Only a reduced bleeding risk by lowering of fibrinogen endures.

The tPA studies [3] showed an initial improvement of $15 \%$ with further improvement over the next 3 months to a total average of $30 \%$. The same can be seen in this study after 10 to 14 weeks. tPA treatment in addition to endovascular treatment shows improvement by $6 \%$ of favorable outcome and lowering by $11 \%$ of unfavorable outcome.

In comparison to the ESCAPE study [8], there is a slightly different outcome in this study. In ESCAPE, $29 \%$ showed a favorable outcome after 90 days with tPA treatment in comparison to $54 \%$ that received additional endovascular treatment. The mean NIHSS is very similar in both groups with 16.5 in the ESCAPE study and 15.7 in this study. A major difference is the randomization of patients. Patients in ESCAPE ranged from age 60 to 81, in this study from 28 to 89 with respect to higher numbers of patients above 80 years of age (25\%). MR. CLEAN [9] included patients from the age of 54 to 76 . The intervention group shows a beneficial outcome for $33 \%$ of patients in the follow-up after 90 days, equal to the outcome in this study with respect to lower average patient age. SWIFT PRIME [10] included 196 patients for both groups with an average age of 65.6. In this study, the average age is 69.5 years and by that close to the average age of ESCAPE with 70.5 years (excluding younger than 60 and older than 81 ) and by average 5 years higher than MR. CLEAN and SWIFT PRIME. The outcome regarding $\mathrm{MRS}$ in SWIFT PRIME shows for thrombolytic treatment a favorable outcome of $36 \%$ after 90 day, which is a better outcome than in this study after tPA treatment. These numbers are higher than in the TPA studies [3]. $60 \%$ reach a favorable outcome after additional endovascular treatment. This is nearly $25 \%$ more than in this 
study. The outcome in this study is equal to the results from the Netherlands by MR. CLEAN. In a more optimal cohort like SWIFT PRIME the results differ dramatically for tPA as well as tPA+EVT. The real-life data in this study includes a wider range of patients with more elderly patients and younger patients with lesser collateral blood supply. After analyzing patients regarding the objective $\mathrm{mRS}$ score, there was an additional questionnaire for all patients after 10-14 weeks. Out of 107 patients, 102 were available for follow up. Patients receiving direct treatment by EVT with revascularization and only minor or no stroke on the follow up CT showed the best outcome in all categories. Patients with the same favorable outcome (mRS 0-2) after prior tPA treatment show a similar result but have slightly higher rates for pain and depression/anxiety. Nearly all patients receiving both treatments were above the age of 60 , while in the EVT group, a third of the patients were aged 30-50. This reflects the different judgement in the EQ5D. Specifically the score for "general ability" was in favor for thrombolysis prior to endovascular treatment. The reason might be found in the different aging of the group where a pensioner might see his abilities in context to his age, while the on average younger patients in the direct EVT group often still need to work and are rendered unable to provide for their families following the stroke. Another aspect is the higher rate

\section{References}

1 Goldstein LB, Bushnell CD, Adams RJ, Appel LJ, Braun LT, et al. (2011) Guidelines for the primary prevention of stroke: A Guideline for Healthcare Professionals from the American Heart Association/ American Stroke Association. Stroke 42: 517-584.

2 Riedel $\mathrm{CH}$, Zimmermann $\mathrm{P}$, Jensen-Kondering $\mathrm{U}$, Stingele $\mathrm{R}$, Deuschl $G$, et al. (2011) The importance of size: Successful recanalization by intravenous thrombolysis in acute anterior stroke depends on thrombus length. Stroke 42: 1775-1777.

3 Sit SP, Bose A, Hirsch JA, Lev MH, Grb FS (2014) Stroke: Implications for IA Therapy. Stroke 44: 3553-3556.

4 Nogueira RG, Lutsep HL, Gupta R, Jovin TG, Albers GW, et al. (2012) Trevo versus Merci retrievers for thrombectomy revascularisation of large vessel occlusions in acute ischaemic stroke (TREVO 2): A randomised trial. The Lancet 380: 1231-1240.

5 Powers WJ, Rabinstein A, Ackerson T, Adeoye OM, Tirschwell DL (2018) 2018 Guidelines for the early management of patients with acute ischemic stroke. Stroke 49: e46-e99. of anxiety and depression in this age group which might cause a worse estimation of the subjective status. This could lead to lower motivation for physiotherapy and progress after the stroke event. Anxiety is a common problem in stroke patients, also after effective treatment. Up to $30 \%$ are affected [11]. This study did not put contrast on the treatment for anxiety and depression. Light needs to be put on in further studies.

\section{Conclusion}

Regarding this study, there are currently no recommendations to switch treatment for patients with proximal MCA occlusion. Patients can improve directly under thrombolysis therapy and by failure still undergo rescue endovascular treatment with a similar outcome to direct EVT after 10-14 weeks. Regarding analysis for quality of life, direct EVT is favorable. Patients eligible for thrombolysis should receive tPA. After insufficient improvement, there is nearly equal benefit with rescue endovascular treatment. This real-life study supports the latest guidelines by the AHA regarding the best medical treatment outcome.

\section{Funding}

The authors received no financial compensation for the study. There was no sponsoring involved.

6 Women P, Cancer EB (2006) A randomized trial of intraarterial treatment for acute ischemic stroke. OA Library 2747-2757.

7 Katz JM, Gobin YP (2006) Merci retriever in acute stroke treatment. Expert Review of Medical Devices 3: 273-280.

8 Goyal M, Demchuk AM, Menon BK, Eesa M, Rempel JL, et al. (2015) Randomized assessment of rapid endovascular treatment of ischemic stroke. New England Journal of Medicine 1019-1030.

9 Berkhemer OA, Fransen PSS, Beumer D, Van den Berg LA, Lingsma $\mathrm{HF}$, et al. (2014) A randomized trial of intraarterial treatment for acute ischemic stroke. New England Journal of Medicine 372.

10 Saver JL, Jahan R, Levy El, Jovin TG, Baxter B, et al. (2012) Solitaire flow restoration device versus the Merci Retriever in patients with acute ischaemic stroke (SWIFT): A randomized, parallel-group, noninferiority trial. The Lancet 380: 1241-1249.

11 Sun Y, Liang Y, Jiao Y, Lin J, Qu H, et al. (2017) Comparative efficacy and acceptability of antidepressant treatment in post-stroke depression: A multiple-treatments meta-analysis. BMJ Open 7: e016499. 